\title{
Ectoparasitos e helmintos intestinais em Felis catus domesticus, da cidade de Lages, SC, Brasil e aspectos sócioeconômicos e culturais das famílias dos proprietários dos animais
}

\author{
Ectoparasites and intestinal helminths in Felis catus domesticus from Lages city, SC, Brazil \\ and social-economical and cultural aspects of owners of family pets \\ Fernanda Magalhães Stalliviere ${ }^{1 *}$; Valdomiro Bellato ${ }^{2}$; Antonio Pereira de Souza ${ }^{2}$ Amélia Aparecida Sartor ${ }^{2}$; \\ Anderson Barbosa de Moura ${ }^{2}$; Luciana Dalla Rosa ${ }^{3}$

\begin{abstract}
${ }^{1}$ Curso de Pós-Graduaçáo em Ciências Veterinárias, Centro de Ciências Agroveterinárias, Universidade do Estado de Santa Catarina - UDESC
${ }^{2}$ Departamento de Medicina Veterinária, Centro de Ciências Agroveterinárias, Universidade do Estado de Santa Catarina - UDESC

${ }^{3}$ Bolsista de Iniciação Científica, Departamento de Medicina Veterinária, Centro de Ciências Agroveterinárias, Universidade do Estado de Santa Catarina - UDESC
\end{abstract}

Recebido em 27 de Março de 2009

Aceito em 19 de Maio de 2009

\section{Resumo}

Os objetivos da realização deste trabalho foram determinar a prevalência de ectoparasitos e parasitos gastrointestinais (GI) em gatos domiciliados, nas regiôes central e periférica, da cidade de Lages, SC e também caracterizar as famílias proprietárias de gatos quanto as suas condiçóes sócioeconômicas e culturais. No período de dezembro de 2005 a dezembro de 2006, foram distribuídos 600 questionários a proprietários de animais cidade de Lages, SC e coletados ectoparasitos e amostras de fezes. A prevalência para ectoparasitos foi de 13,8\% (28/203) e para parasitos GI, 37,8\% (42/111). A prevalência de parasitos em gatos domiciliados da região periférica foi maior que os da região central. Os ectoparasitos observados foram Ctenocephalides felis felis, C. canis e Ctenocephalides híbrido (C. felis felis $\times$ C. canis). Os ovos de helmintos intestinais identificados nas fezes de gatos foram Ancylostoma spp., Toxocara sp., Trichuris sp., família Taeniidae e Oncicola sp. Na região periférica, o maior número de animais positivos pertencia a proprietários de baixa renda salarial e pouca escolaridade. As populaçóes residentes nos bairros da região periférica encontram-se mais expostas aos agentes com potencial zoonótico.

Palavras-chave: Parasitos, gatos domiciliados, diagnóstico.

\begin{abstract}
The purpose of this research was to determinate the prevalence of ectoparasites and gastrointestinal (GI) parasites in domiciliated cats, from central and peripheral region, of Lages city, Santa Catarina State and also characterize the economic, social and cultural status of families' pet owners. From the period of December 2005 to December 2006, 600 questionnaires were distributed to pet owners of Lages city and ectoparasites and faeces were collected. The prevalence of ectoparasites was $13.8 \%$ (28/203) and for GI parasites was 37.8\% (42/111). The prevalence of parasites in domiciliated cats from the peripheral region were bigger than in the central region. The ectoparasites observed were Ctenocephalides felis felis, $C$. canis and Ctenocephalides hybrid (C. felis felis $\mathrm{x} C$. canis). The intestinal helminths eggs observed in cats faeces were Ancylostoma spp., Toxocara sp., Trichuris sp., Taeniidae family and Oncicola sp. In the peripheral region, the largest number of positive animals belonged to owners of low-income wage and low education The peripheral region population are over exposed to potencial zoonotic agents.
\end{abstract}

Keywords: Parasites, domiciliated cats, diagnosis.

\footnotetext{
*Autor para correspondência: Fernanda Magalhães Stalliviere 


\section{Introdução}

Animais de estimaçáo, particularmente cáes e gatos, desempenham importante papel na sociedade. Em muitos lares são importantes como companhia, contribuindo para o desenvolvimento emocional, social e físico de crianças, e no bem estar dos proprietários, particularmente idosos (ROBERTSON et al., 1990; WONG et al., 1999). Apesar dos benefícios, esses animais podem ser acometidos por ecto e endoparasitos e propagar, segundo Rey (2001), agentes responsáveis por zoonoses como larva migrans cutânea e larva migrans visceral.

Os dados de literatura evidenciam a importância dos pulicídeos, como ectoparasitos em gatos, em especial a subespécie Ctenocephalides felis felis. Assim, Fernandes et al. (1996) avaliaram a prevalência de pulicídeos em gatos atendidos no Hospital Veterinário da Universidade Federal Rural do Rio de Janeiro e em clínicas particulares da cidade do Rio de Janeiro, onde constataram 68,8\% de positividade para C. felis felis, enquanto Mendes-de-Almeida et al. (2007), no jardim zoológico, prevalência em gatos para $C$. felis de 38,6\% em 2002; 72,3\% em 2003 e de 42,2\% em 2004. Castro e Rafael (2006) obtiveram, em gatos domiciliados da cidade de Manaus, no Estado do Amazonas, uma prevalência de 72,7\% para C. felis felis. Linardi e Nagem (1973) afirmaram que a distribuição das espécies $C$. felis felis e $C$. canis está relacionada a fatores climáticos, em que a ocorrência de $C$. canis está associada a climas mais frios, fato também verificado por Oliveira e Ribeiro (1982/1983), em Porto Alegre, RS, onde constataram infestaçóes por C. canis em caninos, com maior intensidade nos meses mais frios.

Com relação a estudos sobre helmintos, Gennari et al. (1999), em São Paulo, SP, observaram em amostras fecais de gatos domiciliados a ocorrência de $13,37 \%$ para Ancylostoma spp.; $34,22 \%$ para Toxocara cati; $10,69 \%$ para Dipylidium caninum e 4,81\% para Physaloptera spp. Na mesma cidade e em Guarulhos, SP, Ragozo et al. (2002) verificaram, em amostras de fezes de gatos capturados nas ruas, frequência de $31,16 \%$ para T. cati; 8,7\% para Ancylostoma spp.; 1,45\% para D. caninum; 1,45\% para Platynosomum fastosum. Funada et al. (2007), por meio de exames de fezes em gatos domiciliados e atendidos no hospital veterinário da USP, constataram $6,1 \%$ de animais positivos para T. cati e 2,1\% para Ancylostoma spp. Serra et al. (2003), no Rio de Janeiro, RJ, observaram, por meio de exames coprológicos de 65 gatos domiciliados, prevalência de $26,1 \%$ para Ancylostoma sp. e 9,2\% para Toxocara sp. Na mesma cidade, Labarthe et al. (2004), por meio de necropsias, verificaram que a prevalência foi de 89,6\%, sendo para D. caninum 52,6\%; Ancylostoma braziliensis 65,9\%; A. tubaeforme 8,9\%; Toxascaris leonina 11,9\%; T. cati $25,2 \%$ e Physaloptera praeputialis 9,6\%. Lorenzini et al. (2007), examinaram fezes de gatos domiciliados da cidade de Porto Alegre, RS, e constataram prevalência para Toxocara spp. de 5,9\%; Ancylostoma spp. 3,8\%; D. caninum 1,4\%; Strongyloides spp. 1,7\%; Taenia spp. 0,7\% e T. leonina. 0,4\%

$\mathrm{O}$ aumento da população de gatos influencia na propagação de agentes patogênicos ao homem e a outros animais. Mc Glade et al. (2003) afirmaram que para cada gato a mais no domicílio, o risco de parasitismo aumenta 1,3 vezes. No Estado de Sáo Paulo, foram realizados estudos sobre a proporçáo homem/gato, por Dias et al. (2004), que verificaram a razão entre a população humana e a felina de 30,57:1 e concluíram que a adoção de uma metodologia de estimativa populacional canina e felina domiciliada, baseada em indicadores populacionais humanos, é a mais indicada e facilmente exequível quando comparada ao censo animal e Alves et al. (2005) que constataram em 41 municípios do interior de São Paulo, que a relaçáo entre a população humana e felina foi de 16,4:1 e, que o conhecimento do tamanho da populaçáo felina, permite maior efetividade no planejamento e na avaliação dos resultados de açôes desenvolvidas no sentido da proteção e preservação da saúde dos homens e dos animais. Trabalhos para avaliar o grau de contaminação ambiental parasitária foram realizados por Coelho et al. (2001) que coletaram amostras de solo de 30 praças de Sorocaba, SP, e verificaram que mais de 50\% destas estavam contaminadas com ovos de Toxocara spp. Muradian et al. (2005), em São Paulo, SP, verificaram 29,7\% de amostras positivas para Toxocara spp., 16,2\% para Ancylostoma spp., 5,4\% para Ascaris lumbricoides e 5,4\% para Trichuroidea. As áreas mais comprometidas pela presença de Toxocara spp. foram playground e os quintais das casas, ambos com $66,7 \%$.

O presente trabalho foi realizado com os objetivos de determinar a prevalência de ectoparasitos e helmintos intestinais em gatos domiciliados, das regióes central e periférica, da cidade de Lages, SC; caracterizar as famílias proprietárias dos gatos quanto as suas condições sócioeconômicas e culturais e conhecer a proporção das populaçóes humana e felina.

\section{Material e Métodos}

A cidade de Lages está situada no Planalto Serrano do Estado de Santa Catarina, com altitude de $916 \mathrm{~m}$, latitude de $27^{\circ} 48^{\prime} 57^{\prime \prime} \mathrm{S}$ e longitude de $50^{\circ} 19^{\prime} 33^{\prime \prime} \mathrm{W}$ (BPM, 2006). O clima é subtropical com temperatura média de $14,3{ }^{\circ} \mathrm{C}$, máxima de $35^{\circ} \mathrm{C}$ e mínima de $-7,4^{\circ} \mathrm{C}$, com umidade relativa média de $79,3 \%$, apresentando os meses de dezembro a março como os mais quentes (PML, 2007). A população humana aproximada é de 153.582 habitantes, distribuídos em 68 bairros (IBGE, 2000).

Para este estudo, a cidade foi dividida em duas regióes: central e periférica; destas, foram incluídos, por sorteio, 10 bairros, sendo cinco localizados na regiáo central, Sagrado Coração de Jesus, Coral, Centro, Universitário e São Cristóvão; e cinco bairros ou conjunto de bairros na regiáo periférica, Tributo-Guarujá, Nossa Senhora da Penha, Santa Catarina, Popular-Várzea-Habitação e Caroba-Santa Mônica. Na coleta dos dados, foram sorteadas seis ruas em cada bairro e, em cada rua, iniciando-se pelo lado direito, a cada duas residências, a terceira foi visitada para coleta de dados, perfazendo um total de 600 domicílios.

Os dados foram coletados no período de dezembro de 2005 a dezembro de 2006, por meio de entrevista estruturada. Foram considerados critérios de inclusão na pesquisa a concordância em participar da investigação e responder ao questionário formulado, segundo Richardson et al. (1999), Costa e Costa (2001), Demo (1992) e IBGE-PNAD (2004). A faixa salarial (salário mínimo de $\mathrm{R} \$ 300,00)$ e a escolaridade foram os critérios utilizados para avaliação dos aspectos sócioeconômico e cultural dos proprietários. 
Aos entrevistados que possuíam animais, foram esclarecidos os procedimentos para coleta de dados. Após preenchimento de uma ficha clínica para cada animal, foi realizado o exame clínico. Os ectoparasitos presentes no pelo foram coletados de forma manual e/ou com auxílio de pente fino, acondicionados em frascos de vidro, identificados por número de protocolo, e conservados em álcool $70{ }^{\circ} \mathrm{GL}$. Em animais com suspeita de ácaros, realizaram-se raspados da pele, e o material colocado entre duas lâminas até a chegada ao laboratório. Nos animais com suspeita de Otodectes sp. utilizaram-se zaragatoas para coleta do cerúmen.

As amostras de fezes, recentemente eliminadas, foram recolhidas, acondicionadas em sacos plásticos, identificadas, mantidas em caixas de isopor com gelo e transportadas ao Laboratório de Doenças Parasitárias e Parasitologia do Centro de Ciências Agroveterinárias CAV/UDESC, Lages, SC, onde foram mantidas sob refrigeração entre 2 e $8{ }^{\circ} \mathrm{C}$. Nas residências onde náo houve coleta de fezes dos animais, destes foram contabilizados na amostragem para estabelecer a proporção homem/gato.

Os ectoparasitos foram processados e montados entre lâmina e lamínula de acordo com o descrito por Rey (2001). A identificação foi realizada segundo morfologia descrita por Flechtmann (1973), Soulsby (1987), Bicho e Ribeiro (1998), Linardi e Guimarães (2000), com auxílio de microscópio óptico (aumento 100X). As amostras de fezes foram analisadas pelas técnicas parasitológicas de Willis (1921), Gordon e Whitlock (1939) modificada e de Dennis, Stone e Swanson (1954) modificada. A identificação dos ovos de helmintos foi realizada de acordo com o descrito por Soulsby (1987).

As variáveis analisadas foram o número de gatos infestados e infectados, sendo os resultados expressos em porcentual de animais positivos. $\mathrm{O}$ efeito da região da cidade sobre a porcentagem de animais parasitados foi avaliado pelo Teste de $\chi^{2}$. $\mathrm{O}$ efeito da região da cidade, da classe de idade dos animais e da interação entre essas variáveis sobre o número de animais infectados com Toxocara sp. e total de endoparasitos foi analisado por um modelo linear generalizado binomial (KAPS; LAMBERSON, 2004). Nas classes de idade em que não foram encontrados animais infectados com Toxocara sp., atribuiu-se um valor de 0,5 para animais positivos. Foi utilizado o pacote estatístico SAS (SAS Institute, 1999).

O projeto foi aprovado junto à Universidade do Estado de Santa Catarina - UDESC, pelos Comitês de Ética em Pesquisa com Seres Humanos e de Ética em Experimentação Animal.

\section{Resultados e Discussão}

Ectoparasitos: das 600 residências visitadas, em 118 foi observada a presença de gatos, totalizando 203 animais. Somente pulicídeos foram encontrados e identificados. Dos animais examinados, $13,8 \%$ (28/203) foram positivos, sendo seis $(2,9 \%)$ de bairros da região central, e $22(10,8 \%)$ de bairros da região periférica. Considerando-se os gatos examinados da região periférica, o percentual de infestados foi de $17,9 \%$ e, da região central, 7,4\% (Tabela 1). A prevalência da espécie Ctenocephalides felis felis foi $11,3 \%$. Esse porcentual foi menor que os obtidos por Castro e Rafael (2006), na cidade de Manaus, no Amazonas, 72,7\%: por Fernandes et al. (1996), na cidade do Rio de Janeiro
Tabela 1. Número de gatos domiciliados e percentual de positivos para ectoparasitos e helmintos intestinais, por regióes da cidade de Lages, SC, no período de dezembro de 2005 a dezembro de 2006.

\begin{tabular}{lrcrrr}
\hline \multirow{2}{*}{ Regiáo } & \multicolumn{2}{c}{ Ectoparasitos } & & \multicolumn{2}{c}{ Helmintos } \\
\cline { 2 - 3 } \cline { 5 - 6 } & $\mathbf{N}$ & \% positivos & & $\mathbf{N}$ & \% positivos \\
\hline Central & 80 & $7,4 \% \%^{\mathrm{b}}$ & & 43 & $18,6 \%^{\mathrm{b}}$ \\
Periférica & 123 & $17,9 \%^{\mathrm{a}}$ & & 68 & $50 \%^{\mathrm{a}}$ \\
Total & 203 & $13,8 \%$ & & 111 & $37,8 \%$ \\
\hline
\end{tabular}

Médias seguidas de mesma letra nas colunas náo diferem significativamente $(\mathrm{p}>0,05)$.

e municípios vizinhos, 68,8\%: e por Mendes-de-Almeida et al. (2007), na cidade do Rio de Janeiro, que verificaram para C. felis prevalências de 38,6\% em 2002, 72,3\% em 2003 e 42,4\% em 2004. A prevalência da espécie Ctenocephalides canis foi de 0,5\%. Linardi e Nagem (1973), afirmaram que a distribuição das espécies C. felis felis e $C$. canis, está relacionada a fatores climáticos, em que a ocorrência de $C$. canis está associada a climas mais frios. A influência da temperatura também foi verificada por Oliveira e Ribeiro (1982/1983), em Porto Alegre, RS, onde constataram infestações por $C$. canis em caninos, com maior intensidade nos meses mais frios, julho a novembro. Considerando que o presente experimento foi realizado numa região com temperatura média de $14,3{ }^{\circ} \mathrm{C}$ (PML, 2007), o percentual de $0,5 \%$ pode ser considerado baixo, todavia, Brum et al. (1987), na zona sul do Rio Grande do Sul, citaram a espécie $C$. canis parasitando cães, porém, em gatos, identificaram somente $C$. felis. Deve-se considerar ainda que, em coletas realizadas em cães e gatos, em regióes mais quentes, não foi identificada e espécie $C$. canis (FERNANDES et al., 1996; CASTRO; RAFAEL, 2006; MENDES-de-ALMEIDA et al., 2007). A variação de percentuais constatada na literatura pode estar relacionada, além de fatores climáticos, à concentração de animais e procedência (domiciliados ou não), e a medidas profiláticas, como tratamentos pulicidas. Esses tratamentos foram relatados por 43,8\% dos proprietários da região central e 38,6\% da periférica, sendo possivelmente uma das causas do maior número de gatos infestados na regiáo periférica em relação a central (Tabela 1).

A prevalência de exemplares com atributos morfológicos de C. felis felis e C. canis foi de 2,0\%. Relatos da presença de híbridos foram realizados por autores como Ewing e Fox (1943), Amin (1976). Segundo Linardi e Guimarães (2000), variaçóes da quetotaxia metatibial são comuns em certas regiôes do Brasil onde as duas espécies ocorrem simultaneamente, como constatado no presente trabalho.

Helmintos intestinais: dos 203 gatos computados, foram coletadas amostras de fezes de $111(54,7 \%)$, com prevalência de 37,8\% (42/111) para helmintos intestinais. Das 43 amostras coletadas de bairros da região central, 18,6\% (8/43) foram positivas, sendo todas para Toxocara sp. e, das 68 de bairros da regiáo periférica, $50 \%(34 / 68)$ apresentaram infecçôes simples ou múltiplas, sendo 35,3\% para Toxocara sp.; 8,8\% para Ancylostoma spp.; 1,4\% para Oncicola sp.; 2,9\% para Ancylostoma spp. e Toxocara sp. e 1,4\% para família Taeniidae, Toxocara sp. e Trichuris sp. Houve diferença estatística $(\mathrm{p}<0,01)$ entre o número de animais positivos nas duas regióes (Tabela 1). Resultados de predominância de Ascaridae 
sobre os demais helmintos estão de acordo com Ogassawara et al. (1986), em SP, que constataram maior prevalência de Ascaridae (22,3\%) sobre Ancilostomatidae (19,5\%); Ragozo et al. (2002), em SP, que verificaram a ocorrência principalmente de $T$. cati (28,26\%) e Ancylostoma spp. (8,7\%); Gennari et al. (1999), em SP, ocorrência de 34,22\% para T. cati, 13,37\% para Ancylostoma spp. Funada et al. (2007) em São Paulo, observaram 6,1\% de animais positivos para T. cati e 2,1\% para Ancylostoma spp e Lorenzini et al. (2007), em Porto Alegre, RS, 5,9\% para Toxocara spp., 0,4\% para T. leonina e 3,8\% para Ancylostoma spp. Para Martínez-Barbabosa (2003), a predominância de $T$. cati, deve-se aos gatos, pois, ao defecar no mesmo lugar, solo, areia ou algum material similar, os ovos permanecem e a forma infectante se desenvolve. Quando os gatos limpam-se após a defecação, podem também se contaminar por ingestão de ovos infectantes. Por outro lado, Labarthe et al. (2004), no RJ, observaram maior prevalência para Ancilostomatidae (A. braziliensis 65,9\% e $A$. tubaeforme 8,9\%) em relação à Ascaridae (T. leonina $11,9 \%$ e $T$. cati $25,2 \%$ ), todavia, os autores afirmaram que a idade pode ter influenciado na prevalência, pois, avaliaram gatos com mais de um ano e em alguns trabalhos foi constatado prevalências maiores de Ascaridae em animais mais jovens (VISCO et al., 1978; OGASSAWARA et al.,1986). Serra et al. (2003), no Rio de Janeiro, constataram prevalência em gatos domiciliados de 26,1\% para Ancylostoma sp., 9,2\% para Toxocara sp. e, em gatos errantes, 60,6\% para Ancylostoma, 28,8\% para Toxocara sp. e $15,2 \%$ para T. leonina. Esses autores trabalharam com gatos de idades variadas, sem definição do número de animais por faixas etárias. Oncicola sp. foi identificado pela primeira vez parasitando gatos em Santa Catarina. A presença de Oncicola canis foi relatada em Leopardus pardalis (jaguatiricas), prevalência de 20\% (3/15), por Pence et al. (2003) nos Estados Unidos da América e por Soulsby (1987) parasitando vários animais entre eles cães e gatos domésticos na América do Sul.

$\mathrm{Na}$ Tabela 2, constam as faixas etárias, número e percentuais de animais positivos para Toxocara sp. Houve diferença estatística $(\mathrm{p}<0,01)$ entre as regióes de procedência dos animais, não havendo interação dessa variável com a classe de idade dos animais $(p>0,05)$. A idade dos parasitados não influenciou na porcentagem de positividade ( $p>0,05)$, fato também constatado por Martínez-Barbabosa et al. (2003), no México; todavia, Ogassawara et al. (1986) observaram percentuais maiores de T. cati em gatos, principalmente até os seis meses de idade $(35,8 \%)$ e atribuíram o fato à eliminação de larvas através do leite materno, favorecendo a infecção logo após o nascimento. Visco et al. (1978) e Mc Glade et al. (2003) também encontraram maior prevalência em animais até seis meses. A faixa etária definida na metodologia dos trabalhos, possivelmente é uma das causas de diferenças de prevalências para infecçóes por Toxocara cati.

Os percentuais de gatos infectados com helmintos intestinais, da região periférica, foram maiores com diferença estatística $(\mathrm{p}<0,01)$, quando comparados com os da região central. Resultados que também podem ser justificados pela utilização de anti-helmínticos nos últimos 12 meses, pois, 55,9\% dos proprietários afirmaram ter medicado os animais e deste percentual, 48,6\% são da região periférica e $66.7 \%$ da região central. Mc Glade et al. (2003), na Austrália, verificaram que, nos últimos 12 meses, 91\% dos gatos domiciliados foram desverminados, evidenciando um maior cuidado com a saúde e prevenção das infecções parasitárias, principalmente quando comparados com os resultados do presente trabalho, em bairros da região periférica.

O número de ovos por grama de fezes (OPG) dos animais da região periférica foi, em média, de 32,3 para Ancylostoma spp., 1,5 para Trichuris sp. e 379,4 para Toxocara sp. Na região central, foi identificado somente o gênero Toxocara, com média de 204,6. O número de OPG fornece informaçóes importantes acerca do grau de contaminaçáo ambiental com reflexo no desenvolvimento de formas infectantes. Coelho et al. (2001) coletaram amostras de solo de 30 praças de Sorocaba, SP, e verificaram que mais de 50\% destas estavam contaminadas com ovos de Toxocara spp. Muradian et al. (2005), SP, verificaram 29,7\% de amostras positivas para Toxocara spp., 16,2\% para Ancylostoma spp., 5,4\% para Ascaris lumbricoides e 5,4\% para Trichuroidea. As áreas mais comprometidas pela presença de Toxocara spp. foram playground e os quintais das casas, ambos com 66,7\%. Sommerfelt et al. (2006), em Buenos Aires, na Argentina, examinaram 465 amostras de fezes de gatos coletadas de espaços abertos de instituiçóes públicas e verificaram 58,3\% de positividade; destas, 61,2\% para Toxocara cati e 14\% para Ancylostoma spp. Os dados de literatura, assim como os do presente trabalho, evidenciam deficiências no controle das parasitoses nos animais, e um importante risco para a populaçáo humana, uma vez que algumas espécies são responsáveis por infecções zoonóticas.

Aspectos sócioeconômicos e culturais: dos gatos positivos para ectoparasitos, $50 \%$ pertenciam a famílias com faixa salarial de dois a quatro salários e destes, 78,6\% eram da região periférica. Para helmintos intestinais, $59,5 \%$ dos gatos positivos pertenciam

Tabela 2. Porcentagem de gatos domiciliados positivos para Toxocara sp., das regióes central e periférica da cidade de Lages, SC, em relação à idade, no período de dezembro de 2005 a dezembro de 2006.

\begin{tabular}{|c|c|c|c|c|c|}
\hline \multirow{2}{*}{ Idade (meses) } & \multicolumn{2}{|c|}{ Regiáo central* } & \multicolumn{2}{|c|}{ Regiáo periférica* } & \multirow{2}{*}{ Média } \\
\hline & $\mathbf{N}^{\circ}$ & $\%$ & $\mathbf{N}^{\circ}$ & $\%$ & \\
\hline 0 a 12 & 12 & $33,3 \%$ & 25 & $44 \%$ & $38,6 \%$ \\
\hline 13 a 36 & 10 & $30 \%$ & 29 & $44,8 \%$ & $37,4 \%$ \\
\hline Acima de 36 & 20 & $5 \%$ & 11 & $36,3 \%$ & $20,6 \%$ \\
\hline Média & & $22,7 \%^{\mathrm{b}}$ & & $41,7 \%^{\mathrm{a}}$ & \\
\hline
\end{tabular}

Médias seguidas de mesma letra minúscula nas linhas e maiúsculas nas colunas não diferem significativamente (p > 0,05). *Em quatro animais não foi possível obter dados de faixa etária. 
a famílias com faixa salarial de dois a quatro salários e destes, $88 \%$ estavam na regiāo periférica. Dos gatos positivos para ectoparasitos, $75 \%$ pertenciam a proprietários com ensino fundamental e destes, $85,7 \%$ eram da região periférica. Para helmintos intestinais, 55\% pertenciam a proprietários com ensino fundamental e destes, 90,9\% residiam na região periférica do município. É possível que tanto as condiçóes socioeconômicas quanto culturais influenciaram nos resultados, todavia, náo foi possível avaliar estatisticamente a influência dessas variáveis, devido ao elevado percentual de exames negativos dentro das faixas salariais e das escolaridades pesquisadas.

Proporção entre população humana e felina: nas 600 residências, foi constatada a presença de 2.187 pessoas, média de 3,6 pessoas/domicílio, dados que estão de acordo com a população urbana do município de Lages, SC. (IBGE, 2000). Nas residências, foi verificada a presença de 203 gatos, média de 0,34, valor superior ao encontrado por Dias et al. (2004), em Taboão da Serra, SP, $(0,14)$ e inferior ao verificado por Alves et al. (2005) em 41 municípios do interior de São Paulo $(1,8)$. A proporção pessoa/gato, na cidade de Lages, SC, foi de 10,7:1. Dias et al. (2004) verificaram que a razão entre a população humana e a felina foi de 30,57:1 e Alves et al. (2005) de 16,4:1. Estes autores salientaram que o conhecimento do tamanho da população felina permite maior efetividade de planejamento e de avaliação dos resultados de ações desenvolvidas no sentido da proteção e preservação da saúde dos homens e animais. Embora, descrevendo sobre cães e gatos errantes, Alves et al. (2005) e Ragozo et al.(2002) sugerem que fatores como a quantidade desses animais, pode estar relacionada a transmissão, bem como a manutenção de agentes patogênicos no ambiente mantendo elevada a incidência de enfermidades nos animais que transitam por estes locais, o que representa também um problema de saúde pública.

Considerando-se a população urbana da cidade de Lages, SC, de 153.582 habitantes (IBGE, 2000), e a proporção pessoa/gato $(10,7: 1)$, foi estimada para a cidade de Lages, SC, uma população de 14.353 gatos domiciliados.

\section{Conclusóes}

A maior prevalência de parasitoses ocorre em gatos de proprietários que vivem em bairros da região periférica.

A população humana residente nos bairros da região periférica encontra-se mais exposta aos agentes com potencial zoonótico.

\section{Referências}

ALVES, M. C. G. P. et al. Dimensionamento da população de cáes e gatos do interior do Estado de São Paulo. Revista de Saúde Pública, v. 39, n. 6, p. 891-897, 2005.

AMIN, O. Host associations and Seasonal occurrence of fleas from southeastern Wisconsin mammals, with observations on morphologic variations. Journal of Medical Entomology, v. 13, n. 2, p. 179-192, 1976.

BIBLIOTECA PÚBLICA MUNICIPAL "Carlos Dorval Macedo". Município de Lages. Lages, 2006. (compilado).
BICHO, C. L.; RIBEIRO, P. B. Chave Pictórica para as principais espécies de Siphonaptera de importância médica e veterinária, no Brasil. Revista Brasileira de Parasitologia Veterinária, v. 7, n. 1, p. 47-51, 1998.

BRUM, J. G. W. et al. Artrópodos parasitos dos animais domésticos da zona sul do Estado do Rio Grande do Sul, Brasil. Arquivos Brasileiros de Medicina Veterinária e Zootecnia, v. 39, n. 4, p. 533-537, 1987.

CASTRO, M. C. M.; RAFAEL, J. A. Ectoparasitos de cães e gatos da cidade de Manaus, Amazonas, Brasil. Acta Amazônica, v. 36, n. 4, p. 535-538, 2006.

COELHO, L. M. P. S. et al. Toxocara spp. eggs in public squares of Sorocaba, São Paulo State, Brazil. Revista do Instituto de Medicina Tropical de Sáo Paulo, v. 43, n. 4, p. 189-191, 2001.

COSTA, M. A. F.; COSTA, M. F. B. Metodologia da pesquisa: conceitos e técnicas. Rio de Janeiro: Interciência, 2001. 136 p.

DEMO, P. Metodologia científica em ciências sociais. 2 ed. São Paulo: Atlas, 1992. 287 p.

DENNIS, W. R.; STONE, V. M.; SWANSON, L. E. A new laboratory and field diagnostic test for fluke ova in feces. American Veterinary Medical Association, v. 124, n. 922, p. 47-50, 1954.

DIAS, R. A. et al. Estimativa de populaçóes canina e felina domiciliadas em zona urbana do Estado de São Paulo. Revista de Saúde Pública, v. 38, n. 4 , p. 565-570, 2004.

EWING, H. E.; FOX, I. The fleas of North America. United States: Miscellaneous Publications, 1943. 191 p. (n. 500).

FERNANDES, C. G. N. et al. Pulicídeos de cáes e gatos da cidade do Rio de Janeiro (RJ, Brasil) e municípios vizinhos. Revista Universidade Rural, v. 18, n. 1-2, p. 115-118, 1996. (Série Ciência Vida).

FLECHTMANN, C. H. W. Ácaros de importância médico veterinária. Sáo Paulo: Nobel, 1973. 192 p.

FUNADA, M. R. et al. Frequência de parasitos gastrintestinais em cães e gatos atendidos em hospital-escola veterinário da cidade de São Paulo. Arquivo Brasileiro de Medicina Veterinária e Zootecnia, v. 59, n. 5, p. 1338-1340, 2007.

GENNARI, S. M. et al. Ocorrência de protozoários e helmintos em amostras de fezes de cães e gatos da cidade de São Paulo. Brazilian Journal of Veterinary Research and Animal Science, v. 36, n. 2, p. 87-91, 1999 .

GORDON, H.; WHITLOCK, H. V. A new technique for counting nematode eggs faeces. Journal of the Council for Scientific and Industry Organization, v. 12, n. 1, p. 50-52, 1939.

INSTITUTO BRASILEIRO DE GEOGRAFIA E ESTATÍSTICA-IBGE. Censo demográfico 2000: cidades. Brasília, 2007. Disponível em: <www. ibge.gov.br>. Acesso em: 20 de Janeiro de 2007.

INSTITUTO BRASILEIRO DE GEOGRAFIA E ESTATÍSTICA-IBGE. Pesquisa nacional por amostra de domicílios 2004. Brasília, 2004.

KAPS, M.; LAMBERSON, W. R. Biostatistics for animal science. London: CABI Publishing, 2004. 445 p.

LABARTHE, N. et al. A survey or gastrointestinal helminths in cats of metropolitan region of Rio de Janeiro, Brazil. Veterinary Parasitology, v. 123, n. 1-2, p. 133-139, 2004

LINARDI, P. M.; GUIMARÃES, L. R. Sifonápteros do Brasil. São Paulo: Museu de Zoologia da USP; FAPESP, 2000. 291 p. 
LINARDI, P. M.; NAGEM, R. L. Pulicídeos e outros ectoparasitos de cães em Belo Horizonte e municípios vizinhos. Revista Brasileira de Biologia, v. 33, n. 4, p. 529-538, 1973.

LORENZINI, G.; TASCA, T.; CARLI, G. A. Prevalence of intestinal parasites in dogs and cats under veterinary care in Porto Alegre, Rio Grande do Sul, Brazil. Brazilian Journal of Veterinary Research Animal Science, v. 44, n. 2, p. 137-145, 2007.

MARTÍNEZ-BARBABOSA, I. et al. The prevalence of Toxocara cati in domestic cats in México city. Veterinary Parasitology, v. 114, n. 1, p. 43-49, 2003.

McGLADE, T. R. et al. Gastrointestinal parasites of domestic cats in Perth, Western Australia. Veterinary Parasitology, v. 117, n. 4, p. 251-262, 2003.

MENDES de ALMEIDA, F. et al. Follow-up of the health conditions of an urban colony of free-roaming cats (Felis catus Linnaeus, 1758) in the city of Rio de Janeiro, Brazil. Veterinary Parasitology, v. 147, n. 1-2, p. 9-15, 2007.

MURADIAN, V. et al. Epidemiological aspects of Visceral Larva Migrans in children living at São Remo Community, São Paulo (SP), Brazil. Veterinary Parasitology, v. 134, n. 1-2, p. 93-97, 2005.

OGASSAWARA, S. et al. Prevalência de endoparasitas em gatos na cidade de São Paulo. Revista da Faculdade de Medicina Veterinária e Zootecnia da Universidade de Sáo Paulo, v. 23, n. 1, p. 39-46, 1986.

OLIVEIRA, C. M. B.; RIBEIRO, P. B. Espécies de pulgas que parasitam cães em Porto Alegre e suas prevalências mensais. Arquivos da Faculdade de Veterinária da UFRGS, v. 10-11, n. 1, p. 29-33, 1982.

PENCE, D. B.; TEWES, M. E.; LAACK, L. L. Helminths of the Ocelot from Southern Texas. Journal of Wildlife Diseases, v. 39, n. 3, p. 683-689, 2003.

PREFEITURA MUNICIPAL DE LAGES - PML. Perfil da cidade de Lages... Lages, 2007. Disponível em: <www.lages.sc.gov.br/cidade/perfil. php>. Acesso em: 20 de Janeiro de 2007.
RAGOZO, A. M. A. et al. Ocorrência de parasitos gastrintestinais em fezes de gatos das cidades de Sáo Paulo e Guarulhos. Brazilian Journal of Veterinary Research and Animal Science, v. 39, n. 5, p. 244-246, 2002.

REY, L. Parasitologia: parasitos e doenças parasitárias do homem nas Américas e na África. 3 ed. Rio de Janeiro: Guanabara-Koogan. 2001. $856 \mathrm{p}$.

RICHARDSON, R. J. et al. Pesquisa social: métodos e técnicas. 3 ed. São Paulo: Atlas, 1999. 334 p.

ROBERTSON, I. D. et al. A survey of pet ownership in Perth. Australian Veterinary Practitioner, v. 20, n. 4, p. 210-213, 1990.

SAS INSTITUTE. SAS/STAT User's Guide 8.0. Cary, 1999.

SERRA, C. M. B.; UCHOA, C. M. A.; COIMBRA, R. A. Exame parasitológico de fezes de gatos (Felis catus domesticus) domiciliados e errantes da regiáo metropolitana do Rio de Janeiro, Brasil. Revista Brasileira de Medicina Tropical, v. 36, n. 3, p. 331-334, 2003.

SOMMERFELT, I. E. et al. Prevalence of Toxocara cati and other parasites in cat's faeces collected from the open spaces of public institutions: Buenos Aires, Argentina. Veterinary Parasitology, v. 140, n. 3-4, p. 296-301, 2006.

SOULSBY, E. J. L. Parasitologia y enfermedades parasitarias en los animales domésticos. 7 ed. México: Interamericana, 1987. 823 p.

VISCO, R. J.; CORWIN, R. M.; SELBY, L. A. Effect of age and sex on the prevalence of intestinal parasitism in cats. Journal of the American Veterinary Medical Association, v. 172, n. 7, p. 797-800, 1978.

WILLIS, H. H. A simple levitation method for the detection of hookworm ova. Medical Journal of Australia, v. 8, p. 375-376, 1921.

WONG, S. K.; FEINSTEIN, L. H.; HEIDMANN, P. Healthy pets, healthy people. Journal of the American Veterinary Medical Association, v. 215, n. 3, p. 335-338, 1999. 